\title{
Resource Partitioning Algorithms in a Programmable Service Grid Architecture
}

\author{
Pieter Thysebaert, Bruno Volckaert, Marc De Leenheer, \\ Filip De Turck, Bart Dhoedt, and Piet Demeester \\ Department of Information Technology, Ghent University - IMEC, \\ Sint-Pietersnieuwstraat 41, B-9000 Gent, Belgium \\ \{pieter.thysebaert, bruno.volckaert\}@intec.ugent.be
}

\begin{abstract}
We propose the use of programmable Grid resource partitioning heuristics in the context of a distributed service Grid management architecture. The architecture is capable of performing automated and exclusive resource-to-service assignations based on Grid resource status/properties and monitored service demand. We present two distinct approaches for the partitioning problem, the first based on Divisible Load Theory and the second built on Genetic Algorithms. Advantages and drawbacks of each approach are discussed and their performance is evaluated using NSGrid. Results show that automated resource-to-service partitioning simplifies scheduling decisions, improves service QoS support and allows efficient computational/network resource usage.
\end{abstract}

\section{Introduction}

As more and more application-types are being ported to Grid environments, an evolution from pure computational and/or data Grids to full-scale service Grids [1] is taking place. A "service Grid" denotes a Grid infrastructure capable of supporting a multitude of application types with varying QoS levels. With widespread Grid adoption also comes the need for automated distributed management of Grids, as the number of resources offered on these Grids rises dramatically. Automated self-configuration/optimization of Grid resource usage can greatly reduce management complexity, and at the same time achieve better resource utilization [2]. In this paper, the focus is on the automated deployment of resource partitioning algorithms, which intelligently (i.e. based on current service needs and Grid status) assign Grid resources (network, computing and data/storage resources) to a particular service class for exclusive use during a specified time frame. In doing so, we wish to improve service class priority support and Grid resource utilisation while at the same time simplifying scheduling decisions. Well-known service-driven Grid scheduling frameworks such as AppLeS [4] and GrADS [5] differ from our approach in that we use a Service Management Architecture which operates independent of the scheduling system and actively monitors application behaviour at runtime.

In order to compare the performance of a service managed Grid versus a nonservice managed Grid we use NSGrid (reported upon in [3]), an ns-2 based Grid 
simulator capable of accurately modeling different Grid resources, management components and network interconnections. More specifically, we evaluated both GA-based and DLT-based resource partitioning strategies, both when network aware and when network unaware scheduling algorithms are used.

The remainder of this paper is structured as follows: section 2 gives an overview of the service management architecture. Section 3 elaborates on the different resource partitioning strategies, while their evaluation in a typical Grid topology is discussed in section 4. Concluding remarks are presented in section 5

\section{Service Management Architecture}

We regard a Grid as a collection of Grid Sites interconnected by WAN links. Each Grid Site has its own resources (computational, storage and data resources abbreviated as CR, SR and DR respectively) and a set of management components, all of which are interconnected by means of LAN links. Management components include a Connection Manager (capable of bandwidth reservation support, and responsible for monitoring available link bandwidth), an Information Service (IS) (storing the properties and status of the registered resources) and a Scheduler. Every resource in our model is given an associated service class property (stored in the Information Services). The basic unit of work in our model is a job, which can roughly be characterised by its length (execute time on a reference processor), required input data, the amount of output data and the service class to which it belongs. Each Grid Site has one or more Grid Portals through which users can submit their jobs. Once submitted, a job gets queued at the Scheduler, which in turn queries both local and foreign ISs for resources adhering to the job's requirements. Once the results of those queries are returned, the Scheduler applies one of its scheduling algorithms and (if possible) selects one or more DRs (for input data), together with one or more SRs (for storing output data) and a CR (for processing). If the scheduling algorithm is network aware, the Connection Manager is queried for information about available bandwidth on paths between resources and, once a scheduling decision is made, attempts to make connection reservations between the selected resources.

A distributed service management architecture was implemented in NSGrid in order to evaluate the performance of different resource partitioning strategies. Each Grid Site has a local Service Manager interacting with the local IS, Connection Manager and Service Monitor. The Service Monitor component monitors local characteristics of each service class; it stores inter-arrival times, I/O data requirements and processing length of jobs. At specified intervals, the Service Monitor sends the collected information to all known foreign Service Monitors, so they can keep their 'foreign service characteristics' information up-to-date.

The Service Manager queries the local Service Monitor for information regarding the different services. When the monitored service characteristics do not differ (with regard to a certain threshold) from the ones used to partition the Grid resources in a previous run, no repartitioning will occur. If this is not the case, or if no partitioning has been done yet, the Service Manager will query the 
ISs for Grid resource properties/status. Once the answer to these queries has been received, one of the resource partitioning algorithms (detailed in section 3) is applied to the resource set, and the resulting solution is sent back to the ISs, which in turn change the service-exclusive attribute of their stored resources.

\section{$3 \quad$ Partitioning Strategies}

Recall that we are trying to partition resources into service class resource pools. A solution to this problem is a mapping from resource to a particular service type, and this for all resources returned from the Service Manager - IS queries. A resource can also be assigned service type ' 0 ', meaning it can be used by any service type. Exhaustively searching for a cost function optimum quickly becomes infeasible, as the number of solutions that needs to be evaluated is $(\# \text { servicetypes }+1)^{\# \text { resources }}$. To find a suitable solution in reasonable time, we used two distinct approaches: one uses Divisible Load Theory while the other uses a Genetic Algorithm to obtain a resource-to-service mapping.

\subsection{DLT-Based Partitioning}

Whenever a Grid reaches a steady state (e.g. a Grid processing a periodic load), stochastic parameters regarding the distributions of job IAT, duration and I/Oneeds can be derived for each Service Type by the Service Monitoring Architecture. These parameters can then be used to fuel an ILP designed to

1. Assign an exclusive Service Type to each Computational Resource.

2. Determine the optimal schedule of the periodic workload over the Grid's resources, taking into account the Service Type assignation.

An approximation used to limit the number of integer variables in this problem is to treat the aggregate workload as arbitrarily divisible (hence the name "Divisible Load Theory") [6]. In this context, values of interest are arrivals $s_{s}$ - the load per time unit arriving at site $s$ and belonging to service type $n, \operatorname{Set}_{n}$ and $S i z e_{n}$ - the datasets available to service type $n$ jobs and their respective sizes. Main decision variables in the problem are $x_{c, n}$ (binary, assigning resource type $n$ to $\mathrm{CR} c$ ) and $\alpha_{i, n}^{c}$ (real-valued, amount of service type $n$ load per time unit processed at CR $c$ which arrived at site $i$ ). Auxiliary variables needed to fulfill routing constraints on the input datasets and generated output data have been dubbed $i n_{n, j}^{l}$ (bandwidth needed on link $l$ for transport of dataset $j$ of service type $n$ ) and out $t_{s}^{l}$ (bandwidth needed on link $l$ for transport of output to SR $s$ ).

Using the Divisible Load approach, the resource-to-service assignation can now be modeled as a cost minimization problem with several classes of constraint:1. The capacity constraints to be observed are

$$
\forall c \in C R \cdot \sum_{i \in \text { Sites }} \sum_{n \in S T} \alpha_{i, n}^{c} \leq C a p_{c}
$$

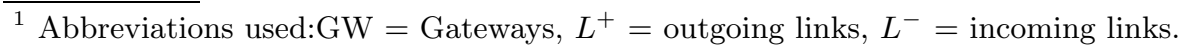




$$
\forall l \in L \cdot \sum_{n \in S T} \sum_{j \in \text { Sets }_{n}} i n_{n, j}^{l}+\sum_{s \in S R} \text { out }_{s}^{l} \leq \text { Cap }_{l}
$$

Network traffic is routed according to following constraints:

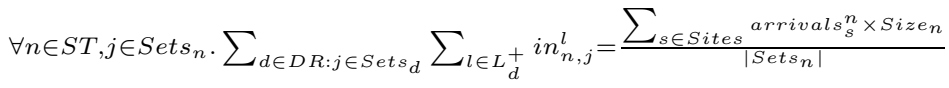

$$
\begin{aligned}
& \forall c \in C R, n \in S T, j \in \operatorname{Sets}_{n} \cdot \sum_{l \in L_{c}^{-}} i n_{n, j}^{l}=\frac{\sum_{i \in \text { Sites }_{i, n}} \alpha^{c} \times \text { Size }_{n}}{\mid \text { Sets }_{n} \mid} \\
& \forall c \in C R, s \in S R . \sum_{l \in L_{c}^{+}} \text {out }_{s}^{l}=\sum_{n \in S T} \alpha_{\text {Site }_{s}, n}^{c} \times \text { Size }_{n} \\
& \forall s \in S R . \sum_{l \in L_{s}^{-}} \text {out }_{s}^{l}=\sum_{n \in S T} \text { arrivals }_{\text {Site }_{s}}^{n} \times \text { Size }_{n} \\
& \forall g \in G W, n \in S T, j \in \operatorname{Set}_{n} \cdot \sum_{l \in L_{g}^{-}} i n_{n, j}^{l}=\sum_{l \in L_{g}^{+}} i n_{n, j}^{l} \\
& \forall g \in G W, s \in S R . \sum_{l \in L_{g}^{-}} o u t_{s}^{l}=\sum_{l \in L_{g}^{+}} o u t_{s}^{l}
\end{aligned}
$$

A feasible schedule is obtained by

$$
\forall i \in \text { sites }, n \in S T \cdot \sum_{c \in C R} \alpha_{i, n}^{c}=\text { arrivals }_{i}^{n}
$$

Constraints concerning the exclusive reservation of each CR:

$$
\begin{gathered}
\forall c \in C R \cdot \sum_{n \in S T} x_{c, n}=1 \\
\forall c \in C R, n \in S T \cdot \sum_{i \in \text { Sites }} \alpha_{i, n}^{c} \leq x_{c, n} \times C a p_{c}
\end{gathered}
$$

The "cost" to be minimized can take on several forms; for instance, the total amount of data traveling over network links per unit of time (in the steady-state Grid) can be described in terms of problem variables as

$$
\sum_{l \in L}\left(\sum_{n \in S T, j \in \operatorname{Sets}_{n}} i n_{n, j}^{l}+\sum_{s \in S R} \text { out } t_{s}^{l}\right)
$$

Using this cost function in the ILP results in a workload schedule and Service Type assignation yielding minimal aggregate network load for a given arrival process. Alternatively, one can choose to minimize the maximal unused CR fraction, which results in an "even" workload distribution across all CRs according to their respective capacities. This can be modeled by adding the constraints

$$
\forall c \in C R, n \in S T \cdot \operatorname{cost} \geq \frac{\left(x_{c, n} \times C a p_{c}-\sum_{i \in \text { Sites }} \alpha_{i, n}^{c}\right)}{C a p_{c}}
$$

\subsection{GA-Based Partitioning}

The resource type assignment can easily be encoded into an $n$-tuple of service type IDs, where $n$ equals the number of resources. These chromosomes can then be fed to a Genetic Algorithm which evaluates the fitness of each chromosome (i.e. possible service type assignment) w.r.t. a cost function (see algorithm 3.1). Unlike an ILP, this cost function need not be "linear" in the decision variables, giving this approach more expressive power than the DLT-based partitioning. 


\section{Algorithm 3.1: Genetic Algorithm(resources)}

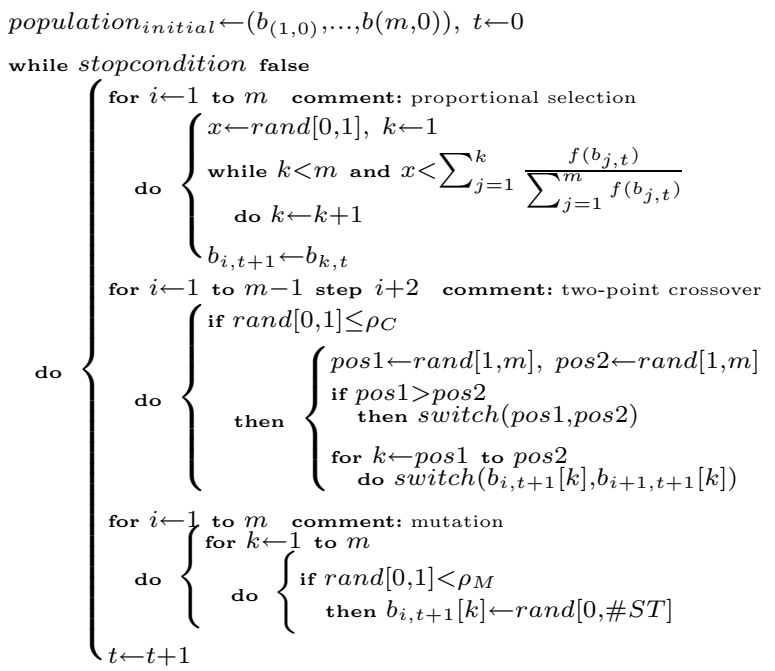

Global Service CR Partitioning. This cost function takes into account the computational processing needs and priority of the different service types $(S T)$. The Service Manager queries the ISs for all local CRs and calculates average service processing needs $\forall S T \cdot$ ppower $_{r e q_{S T}}=$ sites $_{S T} \times \frac{\text { ptime }_{\text {ref }} \text { ST }}{I A T_{S T}}$. Average processing time of a job from service class ST on a reference processor is denoted by time $_{r e f_{S T}}$, while sites $S_{S T}$ denotes the amount of Grid portals launching jobs from this service class. The relative processing power assigned to a service type is then given by: $\forall S T \cdot$ ppower $_{a s g_{S T}}=\sum_{\forall C R \in S T} \frac{\text { speed }_{C R}}{\text { speed }_{C R_{\text {ref }}}} \times$ ptime $_{\text {ref }}$ t $_{S T}$. The importance of assigning resources to foreign service types can be adjusted by the local Service Manager by tweaking the foreign service policy $\rho_{S T_{\text {foreign }}}$. Once CR query answers have been received, GA 3.1 will be started with cost function 3.2 (GA equivalent of equation 13), with an objective to assign each service type a same amount of processing power relative to their requested processing power.

Network Partitioning. Since the Service Monitor keeps track of I/O data characteristics of each service, data intensiveness relative to the other services can be calculated. This in turn can be used to perform per-service network bandwidth reservations. We have implemented a proof-of-concept network partitioning strategy, in which the Service Manager calculates average data re-

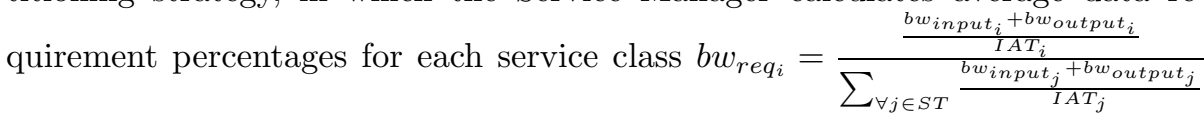
and passes this information to the Connection Manager, which will make service type bandwidth reservations on all network links for which it is responsible. 


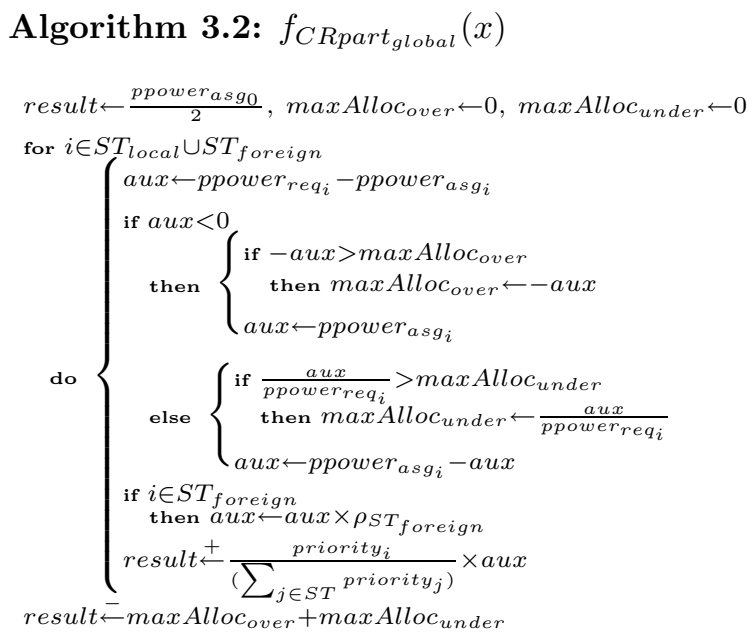

\section{Performance Evaluation}

\subsection{Simulation Setup}

A fixed Grid topology was used for all simulations. First, a WAN topology (containing 8 core routers with an average out-degree of 3) was instantiated. Among the edge LANs, we chose 12 of them to represent a Grid site. Each site's resources and management components are connected through $1 \mathrm{Gbps}$ LAN links, while Grid site interconnections consist of dedicated 10Mbps WAN links. We have assigned $3 \mathrm{CRs}$ to each Grid Site. To reflect the use of different tiers in existing operational Grids, not all CRs are equivalent: the least powerful CR has two processors (operating at reference speed). A second class of CRs has four processors, and each processor operates at twice the reference speed. The third CR type contains 6 processors, each operating at 3 times the reference speed. Conversely, the least powerful CR is 3 times as common as the most powerful $\mathrm{CR}$, and twice as common as the middle one. We have assumed that SRs offer "unlimited" disk space. Each site has at its disposal exactly one such SR. Each site's DR contains 6 out of 12 possible data sets. These data sets are distributed in such a way that $50 \%$ of the jobs can have local access to their needed data.

We used 2 equal-priority service classes (each accounting for half of the total job load); one is more data-intensive, while the other is more CPU-intensive (see table 4.2). Jobs were scheduled using one of two scheduling algorithms; the first algorithm only uses available CR capacities to make decisions, while the second also takes into account network link loads [7]. Once sufficient statistical data about the job parameters had been gathered by the Service Monitor, the Service Manager was instructed to apply a partitioning algorithm to the Grid's resources. We measured average job response time (JRT) and network usage. 


\subsection{Comparison of DLT- and GA-Based Partitioning}

In general, our GA-based partitioning strategy provides more functionality, as it is able to support different priority schemes, shared resource and local vs. foreign service differentiation. Its main drawback is the time needed to complete a GA run (with reasonable results); on our sample scenario, this takes about $800 \mathrm{~s}$, while the DLT-based approach needs only $10 \mathrm{~s}$. For the GA approach, we used Grefenstette's settings, with an initial population of $30, \rho_{C}=0.9$ and $\rho_{M}=0.01$, and a stop condition of 100 runs.

\begin{tabular}{|c|c|c|}
\hline & CPU-Job & Data-Job \\
\hline Input(GB) & $0.01-0.02$ & $1-2$ \\
\hline Output(GB) & $0.01-0.02$ & $1-2$ \\
\hline IAT(s) & $30-40$ & $30-40$ \\
\hline Ref. run time(s) & $100-200$ & $40-60$ \\
\hline
\end{tabular}

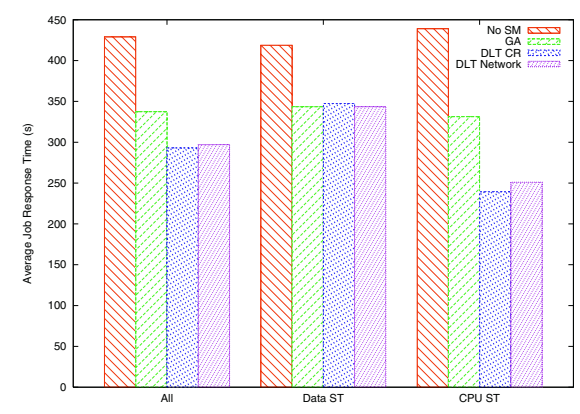

Fig. 1. Job Class Properties \& Non-Network Aware Scheduling: Metrics

\subsection{Non-network Aware Scheduling}

Using a non-network aware scheduling algorithm over all Grid partition strategies results in average JRTs as shown in figure 4.2. Clearly, the use of Grid partitioning based on accurate job characteristic predictions has a positive influence. This behavior is due to resources being reserved for exclusive use by a service class, forcing the scheduler not to assign jobs to less-optimal resources (e.g. non-local access to needed input data), but to keep the job in the scheduling queue until a service-assigned resource becomes available. Optimizing the Grid partitions for minimal network usage does not yield a significant improvement, as the scheduling algorithm does not take into account network loads and diverges from the workload distribution as proposed by the DLT ILP.

\subsection{Network Aware Scheduling}

Values for average JRT and network usage observed when jobs are scheduled using a network aware algorithm are shown in figure 2, Again, when partitioning for optimal CPU usage, average JRTs are improved when compared to JRTs obtained when no partitioning strategy is used2. In analogy, after partitioning for minimal network utilization, network resources are less loaded.

${ }^{2}$ GA stands for GA-based computational partitioning, while GA-CONN denotes GAbased computational partitioning + network partitioning. 


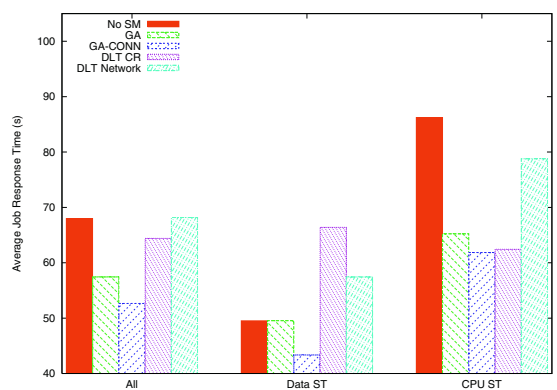

(a) Job Response Time

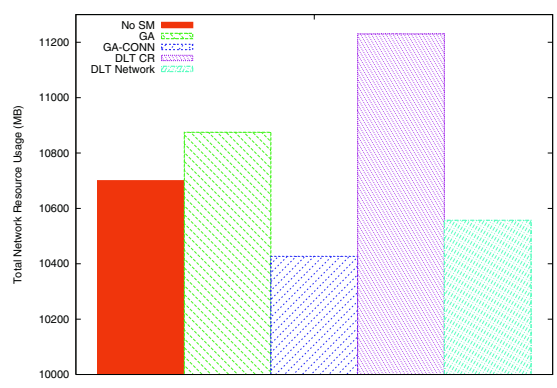

(b) Network Utilization

Fig. 2. Network Aware Scheduling: metrics

We measured the time it takes to calculate a scheduling decision and noticed a decrease in scheduling time of $28 \%$ when comparing the service managed Grid to the non-service managed Grid (i.e. from an average $12.71 \mathrm{~s}$ in the non service managed case to $9.13 \mathrm{~s}$ in the service managed Grid). This can be explained by the fact that when resources are partitioned among services, less resource query results will be returned to the scheduler, allowing easier scheduling decisions.

\section{Conclusions}

In this paper we proposed a distributed service management architecture, capable of monitoring service characteristics at run-time and partitioning Grid resources among different priority service classes. Two specific partitioning algorithms were discussed, and we indicated how our architecture dynamically invokes each algorithm with suitable parameters. We evaluated these algorithms using NSGrid: besides easing the process of schedule making decisions, Service Partitioning does not lead to a deterioration of Grid performance, both when job response times or network resource utilization is measured. A possible preference of one algorithm over the other depends on the trade-off between the size of the needed feature set and the algorithm's computational complexity.

\section{References}

1. I. Foster, C. Kesselman, J.M. Nick, S. Tuecke, "Grid services for distributed system integration", IEEE Computer, Vol. 35-6, pp. 37-46, 2002

2. J.O. Kephart, D.M. Chess, "The vision of autonomic computing", IEEE Computer, Vol. 36-1, pp. 41-50, 2003

3. B. Volckaert, P. Thysebaert, F. De Turck, P. Demeester, B. Dhoedt, "Evaluation of Grid Scheduling Strategies through a Network-aware Grid Simulator", Proc. of PDPTA 2003, Vol. 1, pp. 31-35, 2003 
4. F. Berman et al., "Adaptive Computing on the Grid Using AppLeS", IEEE Transactions on Parallel and Distributed Systems, Vol. 14-4, pp. 369-382, 2003

5. H. Dail, F. Berman, H. Casanova, "A Decoupled Scheduling Aproach for Grid Application Development Environments", Journal of Parallel and Distributed Computing, Vol. 63-5, pp. 505-524, 2003

6. P. Thysebaert, F. De Turck, B. Dhoedt, P. Demeester, "Using Divisible Load Theory to Dimension Optical Transport Networks for Computational Grids", in Proc. of OFC/NFOEC 2005

7. P. Thysebaert, B. Volckaert, F. De Turck, B. Dhoedt, P. Demeester, "Network Aspects of Grid Scheduling Algorithms", Proc. of PDCS 11, pp. , 2004 